\title{
Imaging X-ray sources at a finite distance in coded mask instruments
}

\author{
Immacolata Donnarumma, ${ }^{1, *}$ Luigi Pacciani, ${ }^{1}$ Igor Lapshov, ${ }^{1}$ and Yuri \\ Evangelista $^{1}$ \\ ${ }^{1}$ INAF-IASF Roma, Via Fosso del Cavaliere 100, Rome, Italy \\ ${ }^{*}$ Corresponding author: immacolata.donnarumma@iasf-roma.inaf.it
}

\begin{abstract}
We present a method we developed for the correction of the beam divergence in finite distance sources imaging through coded mask instruments. We discuss the defocusing artifacts induced by the finite distance showing two different approaches to remove such spurious effects. We applied our method to onedimensional coded-mask systems, although it is also applicable to 2D systems as well. We provide a detailed mathematical description of the adopted method and of the systematics introduced in the reconstructed image (e. g. the fraction of source flux collected in the reconstructed peak counts). The accuracy of this method was tested by simulating point-like and extended sources at finite distance with the instrumental set-up of the SuperAGILE experiment, the one dimensional coded-mask X-ray imager on-board the AGILE mission. We obtained reconstructed images of good quality and high source location accuracy. Finally, we show the results obtained by applying this method to real data collected during the calibration campaign of SuperAGILE. Our method was demonstrated to be a powerful tool to investigate the imaging response of the experiment, particularly, the absorption due to the materials intercepting the line of sight of the instrument and the conversion between detector pixel and sky direction.
\end{abstract}

(c) 2018 Optical Society of America

OCIS codes: $340.7430,110.3010$.

\section{Introduction}

"Coded mask aperture imaging technique" is a spatial multiplexing technique to image Xray sources over large fields of view, at X-ray energies where the absorption is dominated by the photoelectric effect, typically below $100-150 \mathrm{keV}$. Although the new multi-layer and long 
focal length telescopes are now starting being used to focus X-rays between 10 and 70-80 $\mathrm{keV}$, this can be only done over a very narrow field of view (below 1 degree) and the coded mask technique is not surpassed yet for imaging large solid angles.

Coded aperture systems encode the sky radiation through a mask of opaque and transparent elements before detector records it. The illuminated mask pattern projects a "shadowgram" on the detection plane shifted with respect to the central position by an amount that depends on the direction towards the point source. The source position in the field of view is reconstructed at a level of accuracy depending on the angular resolution of the system and the number of counts. The experimental parameters of a coded mask system can be optimized to fit the scientific requirements. For example, the angular resolution can be optimized at design level by a proper choice of the size of mask elements and a mask-detector separation. On the other hand coded mask systems have the disadvantage of no direct imaging that implies the need of decoding procedures to reconstruct the sky image. Another disadvantage with respect to direct imaging is that the Poisson noise from any source in the sky is induced in every other position of the sky. We refer the reader to [1] for an extensive and general discussion of the design and properties of coded mask systems. Here we limit our discussion to the properties relevant to the algorithm we have developed.

The image of a source in the sky is obtained by correlating the image accumulated by detector with the proper decoding patterns. Let $D$ be the detector image of the sky, then $\mathrm{D}$ is given by the convolution of the true sky image $S$ with the mask pattern $M$ plus the background $B$ :

$$
D=S \otimes M+B .
$$

It is possible to reconstruct the sky image $S^{\prime}$ by cross correlating the detector image with the reconstruction matrix $\mathrm{R}$, which has to be opportunely defined:

$$
S^{\prime}=D \odot R=S \otimes(M \odot R)+B \odot R .
$$

The reconstruction is obtained when $M \odot R$ is the identity matrix. This can be achieved if the mask is based on a pseudo-random URA (Uniformly Redundant Array) pattern, built using cyclic difference sets for which the auto-correlation function is a $\delta$ function (see [2]). If the mask pattern presents an open fraction of 0.5 (the number of opaque elements equal to the number of the transparent ones) the reconstruction matrix is given by $R i j=1,-1$ for transparent and opaque elements, respectively. It is evident from Eq. (2) that this kind of matrix allows for the background subtraction in the assumption of its uniformity. Generally speaking, if the complete mask pattern is projected on the detector then $S^{\prime}=S$. For several mask-detector systems this is true only for a limited fraction of the field of view, while the remaining sky is only partially coded due to the collimator shadow. In these regions of the 
reconstructed images a source induces false peaks (the so-called "coding noise"). The removal of these false peaks occurs by using an Iterative Removal of the Sources in the sky (IROS). We refer the reader to [3] for details about the removal of such effect from sky images.

Imaging source at finite distance (i. e. divergent beams) is more complicated because the size of the shadow in the detector plane is magnified depending on the distance of the source to the plane itself. In this paper we will call the effect of the beam divergence on the optical system mask-detector as a "defocusing" effect, although this term is not fully appropriate.

Using simple geometrical considerations the magnification $m$ of the mask pattern size is related to the source to the mask plane distance (here $z$ ) and to the mask-detector separation $f:$

$$
m=(z+f) / z .
$$

The standard deconvolution procedure does not allow the source peak reconstruction because the beam divergence defocuses the incident radiation. However we can consider the detector image as the result of convolution of the source with a virtual mask, i. e. the magnified version of the true mask, or alternatively we can consider a virtual detector (the reduced version of the true one) as the convolution with the true mask. Both approaches consist in a sort of a "collimation" of the modulated radiation thus suppressing the defocusing due to the beam divergence. In the case of a virtual mask, the focusing requires the replacement of the reconstruction matrix $R$ in Eq. (2) with a matrix $R^{\prime}$ obtained by expanding $\mathrm{R}$ with a factor of $m$ so that $R^{\prime} i j$ will have values between -1 and 1 , defined by interpolation. Instead, the use of a virtual detector offers the advantage to leave $R$ unchanged. In both cases, the open fraction value (0.5) is altered, because only a fraction of the illuminated mask pattern is projected on the detector.

Thus, there is no strong argument in support of the choice of either procedure. However, in order to have the same configuration of mask-detector system as for parallel beams (i. e. the same correspondence between mask element and detector pixel) and to decode the image with the same reconstruction matrix $R$, we decided to develop a reconstruction method based on a virtual detector "reduced" by a factor equal to $1 / \mathrm{m}$.

The reduction procedure (hereafter "shrinking procedure") has been checked on the 1D coded mask system of a true experiment, SuperAGILE, however this procedure, mainly based on simple geometrical considerations, may also be used for 2-D systems.

In the next section we present the reduction procedure together with a detailed analytical description of the systematics related to the method for the case of 1-D coded maskinstruments.

In section 3 we show the reliability of the method by means of computer simulations, assuming the instrumental set-up that was used to validate our method by means of exper- 
imental tests. The results of these tests are presented in section 4, where our method was applied to the ground calibrations of the SuperAGILE experiment ( [4]).

\section{The shrinking procedure}

The goal of the shrinking procedure is to remove the artifacts in the source image due to the geometrical magnification of the mask shadowgram. As discussed in the previous section, our approach is to demagnify the shadowgram in the detector image by reducing the detector array size. Suppose to have a detector of $r$-elements $D$, by shrinking we mean redistributing all the counts, by linear interpolation, on a new array $\tilde{D}$ ("virtual detector") with dimension $n$ equal to a fraction $1 / m$ of the original size (putting the last $r-n$ elements equal to zero). The perfect suppression of magnification artifacts requires an accurate knowledge of the magnification factor, that is of both source to the mask plane $z$ and detector-mask separation $f$. If these quantities are known, the virtual detector image $\tilde{D}$, corresponding to the correct value of $m$, is created and then the standard cross-correlation with the reconstruction matrix $R$ (see Eq. (2)) is applied.

For a finely segmented detector it is possible to overcome the knowledge of these quantities by developing a procedure that tries to optimize the signal to noise ratio of the source image by altering the value of the magnification factor.

This is done generating a set of virtual detector images (each of them reduced according to a proper value of $1 / m$, "the shrinking factor").

By cross-correlating these detector images with the reconstruction matrix $R$ we generate a set of "sky images", that show more or less blurred peaks at a given position. The shrinking factor corresponding to the highest signal to noise ratio will represent "the best approximation" of the true source image that is also the best we can do ignoring the detector response. On the other hand a maximum likelihood approach can be used to determine e. g. the Point Spread Function of the instrument when our best shrinking factor is found. We do not enter into the details of this approach because we are focused on the removal of the source image artifacts, but we refer to [5] for a description of this procedure.

Right now we consider the signal to noise ratio of the image as a function of one variable, $m$. This is not true for a realistic detector for which the ratio between mask and detector elements size varies typically in the range $[1,3]$. Independently on the infinite or finite distance of the source, the shape of the source peak in the reconstructed images varies depending on the position in a "sky" bin (hereafter "source phase"). If the source is located in the center of a sky bin, i. e. each element of the mask is projected in exactly one detector element, the fraction of detector counts collected in the peak is $100 \%$. Instead, for all the other locations within a sky bin the total counts will be collected on a wider sky bin range, each bin scaled to the fraction of mask element projected in detector pixel ( [3]) . 
Thus, we consider the signal to noise ratio of the source image as a function of one more parameter accounting for the source phase (within the beam divergence) with respect to which photons are collimated. This is expressed by means of the detector pixel intercepted by this direction towards the source. It is assumed as the center of the shrinking procedure (hereafter "the shrinking base point" or $i_{s h r}$ ).

In Fig. 1 we show the value of the peak in the sky image as a function of both the shrinking factor and the shrinking base point. The bi-dimensional maximum shape shows an absolute maximum as a function of the $z$, being a periodic function in the domain of the shrinking base point (see bottom panel in Fig. 1). While it is easy to understand the bidimensional maximum along z-axis, a periodic behavior in the shrinking base point domain is less straightforward to interpret. The maximum along the shrinking factor axis corresponds to the best focusing, and shrinking with the wrong factor leads to defocus, thus loosing an increasing fraction of the peak counts.

The periodic behavior of the maximum as a function of the shrinking base point position can be explained by simple geometrical considerations presented below: a line connecting the source and the edge of a mask element (see Fig.2) that also intercepts the edge of a detector pixel, determines a local maximum in the shrinking base point domain (see left panel of Fig. 2, the short-dashed line passing through the mask element edge represents one of the directions where the shrinking starts). In fact, shrinking with respect to this point effectively means collimating the source incident radiation with respect to the direction of this line (see right panel in Fig. 2). The possible directions for which this happens define the periodicity along the shrinking base point axis. We note that it is more correct to call it a quasi-periodicity, because the peak local maxima could be slightly different depending on the mask pattern intercepted by the corresponding directions.

We now quantify the periodicity in the peak counts, $p$, as function of $z$ and of the beam divergence. Let $i_{s h r}^{\prime \max }$ be a point of local maximum in the shrinking base point domain connected with the edge $e^{\prime}$. The place of points $i_{s h r}^{\max }$ of the local maxima in the shrinking base point domain is determined by the following relation:

$$
\begin{aligned}
& \left(i_{\text {shr }}^{\max }-i_{\text {shr }}^{\prime \max }\right) \times w_{\text {det }}=\left(e-e^{\prime}\right) \times w_{\text {mask }} \times m \\
& \text { or, introducing } n=\left(e-e^{\prime}\right) \text { and } p=\left(i_{\text {shr }}^{\max }-i_{\text {shr }}^{\prime \max }\right) \\
& p \times w_{\text {det }}=n \times w_{\text {mask }} \times(z+f) / z .
\end{aligned}
$$

where $w_{\text {det }}$ and $w_{\text {mask }}$ are the detector pixel and the mask element sizes, respectively. Since $w_{\text {det }}$ and $w_{\text {mask }}$ are proportional, then $p \propto n \times(z+f) / z$, thus $p$ is a rational number that approximates the corresponding magnification factor $m=(z+f) / z$. When the source lies at a distance equal to a multiple of the mask-detector separation, it is easy to determine $p$; 
e. $\mathrm{g}$. for $z=2 f$ the periodicity $p$ is equal to 3 pixels in the detector domain. When $z \rightarrow \infty$ (that is, approaches the infinite distance case) the maximum as a function of the shrinking base point becomes an almost constant function $(p / n \rightarrow 1)$.

We now describe as it is possible to determine either $z$ or $f$ when the best magnification factor is found. This requires that one of the two parameters is known with a reasonable accuracy given the degeneracy present in Eq. (3). For example, if $z$ is ignored, it will be known within an uncertainty equal to $\Delta f /(m-1)(m=1$ is reached for $z \rightarrow \infty)$ where $\Delta f$ includes both the uncertainty introduced by the accuracy of the metrological measurement and the error due to its dependence on energy $(\Delta f(E))$. The efficiency of the detector and the operating energy band require different approaches in the use of shrinking procedure.

Depending on the absorption length of the incident photons, it is possible to have a large spread in the mask-detector separation, causing a systematic error on $f$. If the radiation is preferentially absorbed at a given depth in the detector thickness, one single value of $f$ has to be used with an uncertainty associated with the dispersion in the absorption. In all the other cases we suggest to use the shrinking procedure separately for each value of $f$, taking into account its own error $(\Delta f(E))$.

When $z$ has been determined, it is easy to determine the lateral displacement of the source $y$ by means of the shrinking base point $i_{s h r}^{\max }$ corresponding to a given direction $\phi$. It is defined as $y=(z+f) \times \tan (\phi)+i_{\text {shr }}^{\max } \times w_{\text {det }}\left(w_{\text {det }}\right.$ is the detector element size $)$.

The goal of our method is the deblurring of the source image, that is approximate the infinite distance case. However, even in the best case, the result of our method will show a clear difference with the infinite distance case, due to the systematics introduced by the linear interpolation.

We now quantify the systematics. To this purpose we compare the sky image of a source at infinite distance with that obtained for a point-like source at finite distance, neglecting the source spatial extension.

Assume a source at on-axis position, that is we neglect the flux attenuation correlated with the source phase. As opposite to the infinite distance case, where the sky image peak has to include almost the $100 \%$ of the detector recorded counts, in the finite distance case only a fraction of the recorded counts will contribute to the peak in the sky image, by an amount that depends on the distance $z$ and on the beam divergence.

Let be $D$ a function describing the counts recorded by the detector pixels. Shrinking the detector means to build a new function $\tilde{D}$ that satisfies the relation $\tilde{D}(i)=D(i \times m)$. $D(i \times m)$ is obtained linearly interpolating between the counts of the pixels $[i \times m]$ and $[i \times m]+1(D([i \times m]+1)$ and $D[i \times m]$, respectively) where the square brackets indicate 
the integer part of the number. This implies that

$$
\tilde{D}(i)=(i \times m-[i \times m]) \times(D([i \times m]+1)-D[i \times m])+D[i \times m] .
$$

For simplicity we define $j=[i \times m]$. As it is shown in Fig. 3 the counts $D(j)$ recorded by the $\mathrm{j}$-th detector pixel depend on the value of the 3 elements of the mask pattern $M(j-$ $1), M(j), M(j+1)$ and on the shadowgram produced in the detector. This means that it also depends on the fraction of the flux lost by $D(j-1), D(j), D(j+1)(a, b, c$ in Fig. 3$)$, expressed as a function of the angle $\theta$ in the coding direction (see Fig. 3). Then the same dependence applies to the counts of the virtual detector $\tilde{D}$.

Each element of the mask pattern can have two possible values $(0,1)$, causing $2^{3}$ different combinations of the numerical terns to exist. The virtual detector pixels will then collect a number of counts depending on the distribution of the illuminated numerical terns and on $\theta$ (see Appendix for details).

Finally, a standard cross correlation between mask pattern and virtual detector can be performed, multiplying by -1 the detector counts in the i-th pixel coming from a tern with central element equal to $0, \tilde{D}_{i}^{0}$, and by 1 the counts of detector corresponding to a tern with central element equal to $1, \tilde{D}_{i}^{1}$.

In the assumption that the eight numerical terns are equally distributed (if not the following relation is still valid), we can calculate the total number of counts on the virtual detector by integrating Eq. (4) from the minimum up to the maximum angular divergence for a fixed $z$; the integration in $\theta$ is done over the values of $\theta$ corresponding to all the illuminated portions of the detector.

Then the resulting normalized sky image will be:

$$
S k y_{\text {norm }}=1-2 \times \tilde{D}_{\text {int }}^{0} /\left(\tilde{D}_{\text {int }}^{1}+\tilde{D}_{\text {int }}^{0}\right)
$$

where $\tilde{D}_{\text {int }}^{1}=\int_{\triangle \theta} \tilde{D}^{1} \times f(\theta) d \theta, \tilde{D}_{\text {int }}^{0}=\int_{\triangle \theta} \tilde{D}^{0} \times f(\theta) d \theta$ and $f(\theta)$ is a function accounting for the $\theta$ variation within the beam divergence.

Eq. (5) tells us how much the ratio between peak counts and the total detector counts is affected by the magnification. In particular, it easy to see from Eq. (5) and the tern values described in the Appendix that this fraction increases with $z$ approaching 1 when the source is at infinite distance.

There may be cases for which the dependence on $z$ is negligible. In a mask-detector system, with the ratio between mask element and detector element size equal to 2 , the peak to total counts ratio is expected to not vary with $z$. In this case, the number of terns to be considered is reduced from $2^{3}$ to 4 (only terns with 2 equal contiguous elements have to be considered) and it is possible to verify that Eq. (5) is constant with $z$. 
With respect to the overall shape of the reconstructed sky image, we expect the point spread function to be symmetric because the brightest peak is obtained when the element of the mask pattern element is in phase with one detector strip. A small asymmetry may be present due to the computational accuracy and can be neglected. Consequently we consider any asymmetry in the reconstructed point spread function as associated with real physical and geometrical effects in the instrument.

In the next sections we describe the results achieved applying the shrinking procedure to the imaging with the SuperAGILE experiment with simulations and experimental tests.

\section{Computer simulations of beam divergence effects: SuperAGILE}

To verify the reliability of our method we performed Monte Carlo simulations of a large set of sources imaged by SuperAGILE.

SuperAGILE is the hard X-ray monitor on-board AGILE (Astro rivelatore Gamma a Immagini LEggero, see [6]) operating in the nominal energy range 18-60 keV. It consists of four one-dimensional coded-mask detectors, encoding the same direction in pairs. The collimator and the mask define a single (one-dimensional) field of view of $107^{\circ} \times 68^{\circ}$ for each of the 4 detectors, orientated at $90^{\circ}$ for the two pairs of units.

The masks code is a Hadamard sequence of 787 elements generated with the method of quadratic residue, to which one element has been added to reach the complete symmetry and reversibility of the mask. Each nominal mask element is $242 \mu \mathrm{m}$ wide, twice the dimension of the strips of the silicon micro-strip detector, to ensure the proper sky image reconstruction. For a detailed description of the experiment we refer the reader to [4] and to references therein.

We simulated both point-like sources as well as extended sources, varying their positions in $z$ (source mask-plane distance) and along the plane parallel to the mask to study the accuracy of the focusing method as a function of distance and lateral displacement. The choice of distances from the detector plane is influenced by the aims to be achieved: as an example, tomography is best achieved by imaging source located as close as possible; understanding detector response to infinite distance sources requires going far from the detection plane $(z \rightarrow \infty$ is equivalent to $m \rightarrow 1)$. Imaging sources very close to the detection plane gives rise to important side effects. The radiation intensity reaching the detector is no longer uniformly distributed because its path-length $r^{\prime}$ increases going far from the normal vector $r$ ("inverse square effect"). Moreover, the angular subtense of each hole decreases across the aperture ("obliquity effect") leading to flux attenuation proportional to the same angle. We refer the reader to [7] for details about the inverse square and obliquity effects.

Since we are interested to reach the best focusing of the image, we choose distances for 
which the side effects can be neglected. Thus we varied the distance of the simulated source to the detector plane in the range $100-300 \mathrm{~cm}$ (for SuperAGILE, with its mask-detector distance of $\sim 14.2 \mathrm{~cm}$, this means from $m \sim 1.1$ to $m \sim 1.05)$. On the basis of the reconstructed images and properties such as the peak counts, the point spread function widening and systematic asymmetry of the shape, we found no strong arguments to discriminate among these distances (see columns 1,2 in Table 1).

In particular, the reduction of the peak counts is $\sim 17 \%$ with respect to the source counts recorded on the detector in the range $100-300 \mathrm{~cm}$. This is what we expected from the analytical description of the peak-counts attenuation, because for SuperAGILE the ratio between mask elements and detector element size is equal to 2 (see previous section). As expected, for a source located at infinite distance we did not find any reduction of the peak counts.

The imaging at finite distance also affects the Point Spread Function (see dot line in Fig. 4 for comparison with the infinite distance simulation). A widening of two side lobes (with $\sim 10 \%$ of the recorded counts) appears. The flux attenuation (or the image widening) becomes larger if one considers an extended source: for a monochromatic source at $32 \mathrm{keV}$, with a disk shape $5 \mathrm{~mm}$ in diameter we predict a factor of $\sim 40 \%$ (see dashed line in Fig. 4). It is clear that this further widening depends on the spatial profile of the source.

In the left panel of Fig. 5 we show the image of a simulated point source at $z+f=250 \mathrm{~cm}$ obtained with the standard deconvolution method (see Eq. (2)), i. e. without applying the focusing procedure. The right panel of the same figure shows the significant peak emerging after the shrinking procedure is applied.

By means of these simulations we also studied the error in the source direction parallel to the optical axis associated with statistical fluctuations. We found that the $1-\sigma$ error for the parallel direction $z+f$ is $\simeq 0.1 \mathrm{~cm}$ if a signal to noise ratio of $\sim 300$ is achieved.

The performed simulations also drove set-up of calibrations of the SuperAGILE experiment with radioactive sources in laboratory, given the reconstruction accuracy described above. In particular the choice of the vertical displacement of the source was based on a trade-off between the accuracy on determination of the physical quantities to be calibrated and the integration time needed for these measurements.

For example one of our major goals was to determine the mask-detector separation $f$ from the magnification factor, knowing $z$ (see Eq. (3)), thus calibrating the relation between the detector pixel $i$ and sky direction $\theta_{s k y}^{i}$ by means of the following relation:

$$
\theta_{\text {sky }}^{i}=\operatorname{arctg}\left(i \times w_{\text {det }} / f\right)
$$

In this case the farther the point source is the better the accuracy and the determination of $f$ can be. In particular, assuming a $z+f$ distance of $\sim 200 \mathrm{~cm}$ and taking a reasonable 
accuracy in the experimental measurement of $z$ into account $(\sim 0.1 \mathrm{~cm})$, we predict an accuracy of $\sim 75 \mu \mathrm{m}$ in the estimation of $f$ (see Table 1 , it is $\sim 165 \mu \mathrm{m}$ at $z+f=100 \mathrm{~cm}$ ).

We now see as this error is propagating on the reconstruction of the sky direction (see Eq. (6)). The uncertainty in the reconstruction of the sky direction as a function of $f$ is given by $1 / 2 \times \sin \left(2 \theta_{\text {sky }}^{i}\right) \times \Delta f / f$, then a $\Delta f$ equal to $75 \mu \mathrm{m}$ corresponds for a 30 degree off-axis position to an accuracy of 0.013 degrees, while $\Delta f=165 \mu \mathrm{m}$ corresponds to 0.028 degrees. These numbers have to be compared with the value of $\sim 0.036$ degrees for sky pixel size at 30 degrees. Thus, a distance greater than $200 \mathrm{~cm}$ allows to calibrate the sky direction reconstruction capability with an uncertainty that is well below the pixel size.

We also estimated the error in the direction orthogonal to the optical axis, i. e. the lateral displacement of the source, defined as follows

$$
y=(z+f) \times \operatorname{tg}(\phi)+i_{s h r}^{\max } \times w_{\text {det }}+\triangle
$$

(where $\phi$ indicates the angle chosen for the shrinking, $i_{s h r}^{\max }$ is the point of local maximum along the shrinking base point axis, $w_{\text {det }}$ is the detector strip length and finally $\triangle$ is a correction parameter related to the detector-mask alignment) with Montecarlo we found an accuracy of $|\Delta y| \simeq 0.0045 \mathrm{~cm}$ for a source located at $200 \mathrm{~cm}$, due to statistics. This means that for distances larger than $200 \mathrm{~cm}$ from the detection plane, we would expect an uncertainty of $y$ less than $3 \%$ of detector pixel size.

Therefore, for the ground calibrations of the SuperAGILE experiment we requested a source distance equal or greater than $200 \mathrm{~cm}$. However all the previous discussions assume that we preserve the counts statistics at any distance, and this is of course not true, for a given intensity of the calibrating source. This suggests to not move too far from the 200 $\mathrm{cm}$, in order to avoid divergence of the integration time needed to reach a given statistical accuracy.

\section{Laboratory results}

The shrinking procedure was demonstrated to be a powerful tool to use diverging beam sources at finite distance to calibrate coded-mask systems. In this section we present how the shrinking procedure has been used during the calibrations of the SuperAGILE experiment, currently flying on-board the AGILE space mission (see [5] and [8] for the first calibrations of this instrument performed in 2005).

The final SuperAGILE ground calibrations were performed on January 2007. The experimental setup was studied to locate the radioactive sources at $\sim 200 \mathrm{~cm}$ from the detection plane. The micrometric position of the sources was targeted with a laser tracker with respect to reference points on the spacecraft structure, and to the reference optical cubes aligned to the stars sensors of the AGILE satellite. For this calibrations campaign we used 3 radioactive 
sources: $C d^{109}$ (with a main line at $22 \mathrm{keV}$ ), $I^{125}$ (line complex at 27-32 keV) and $A m^{241}$ (main line at $59.5 \mathrm{keV}$ ). As discussed above, the main goal of the shrinking procedure is to allow the study of the instrument imaging response without using parallel beam sources, and using instead the more easily accessible (quasi) point-like commercial radioactive sources. This implied a large set of measurements covering from one edge of the field of view (FOV) to the opposite one in coding direction as well as in no coding one. The sample of the scanned positions covered the range -50 to +50 degrees with a step of 10 degrees. The complete scanning was performed only for the $C d^{109}$. The other sources were instead used to sample fewer positions in the center of the FOV (see [4]).

In Fig. 6 we present a sample of the zoomed source images for SuperAGILE detector units. In Fig. 7 we show the full source sky image in the Field of View for 0 and 30 degrees off-axis. The quality (quite-symmetric) and the widening of the peak shape agree with what is expected from Montecarlo simulations of a source with similar spectral energy and spatial profile. This is corroborated by the values of the distance $z+f$ inferred by the magnification factors obtained for each measurement. They are in agreement (within the uncertainties of $\sim 1-2 \mathrm{~mm}$ ) with the distances measured with the laser tracker.

Then we can say that the correction works well for each of the off-axis positions showing as it is influenced only by the statistics (similar in all measurements). Looking at the last two images, at $\sim 40,50$ degrees, the correction appears to work fine, as demonstrated by the symmetric shape of the point spread function, but a distortion of the baseline is also found. A deeper analysis of this feature showed the presence of a small "leak" in the collimator shielding affecting the image of this detection unit for angles greater than 20 degrees.

Given the level of accuracy achieved in the corrected source images, it has been possible to use the detector images reduced by the correct magnification factors as well as the reconstructed sky images for calibration purposes. In particular we used the first ones to estimate the transparency of the materials intercepting the line of sight of SuperAGILE. On the other hand the sky images has been used to calibrate the pixel-angle conversion with the purpose to obtain an analytical law.

This has been done associating the centroid position of the reconstructed images with the angles provided by metrological measurements (triangle points in Fig. 8). The simplest relation that connects pixel and angle is provided by Eq. (6). However we found that a best fit to the data is found by adding a geometrical offset between mask and detector planes plus a pixel offset $\Delta$ to Eq. (6).

In fact, a better fit to the data (solid line in Fig. 8) has been obtained using the following parametric function: 


$$
\theta_{s k y}^{i}=\operatorname{arctg}\left[\left(m_{s t}+(i+\triangle) \times w_{\text {det }}\right) / f\right]
$$

where $m_{s t}, w_{\text {det }}$ and $f$ are the distance of the first mask element in the SuperAGILE reference system, the size of pixel and the mask-detector separation, respectively. As evident in residuals (bottom panels in Fig.8) the agreement between measured angles and those inferred by the best fit is quite good, being $\leq 2$ arc minutes (remind for comparison that SuperAGILE spatial resolution is 6 arc minutes).

We stress as the understanding of such effect is crucial in the procedure of Iterative Removal of Sources (IROS) in coded mask instrument. In fact this requires a good understanding of the instrumental response to a source in a given position in the sky.

\section{Conclusions}

We described the method developed to correct for the beam divergence in coded mask imaging of sources placed at finite distance from the detector plane. The method has been studied and applied on 1-D coded-mask instrument, although its extension to the 2-D instruments is feasible. It works to collimate the modulated radiation from the X-ray source with reference to one direction within the beam divergence. The choice of the direction is determined by the best suppression of defocusing effects of the image, associated to the magnification of the source shadowgram in the detector.

This requires the knowledge of the source distance to the mask plane and the mask-detector separation. If these two values are not known or known within some uncertainty, the best focusing may be found searching for the highest signal to noise ratio in the reconstructed image. This is a function of both the source distance to the detector plane (and then of the magnification factor) and the direction (within the beam divergence) with respect to which photons will be collimated. We argued that there is only a value of the magnification factor corresponding to the maximum of the signal to noise ratio, while there are several (depending on the distance) favorite directions to collimate photons, going to one possibility for the infinite distance case. A detailed study of the systematics introduced by our method on the reconstructed source image has been done. We found a reduction of the peak counts

compared with the infinite distance, which amount depends on the source distance and the beam divergence (neglecting the source spatial extension).

To check the reliability of the method we performed a set of Monte Carlo simulations of X-rays sources imaged by the SuperAGILE experiment.

We simulated point-like sources as well as extended sources at finite distance. We found that the flux attenuation with respect to the parallel beam case mainly depends on the geometric extension of the source. The dependence on the source distance is quite negligible due to the particular configuration of the coded-mask system of SuperAGILE. 
Then we found a flux attenuation of $\sim 17 \%$ compared with the parallel beam case for a point-like source. This clearly quantifies the widening of the Point Spread Function due to the systematics of our method. The flux reduction increases to $\sim 40 \%$ if e. g. a source with a disk shape of $5 \mathrm{~mm}$ in diameter is considered.

By means of these simulations, we estimated the statistical errors on both orthogonal and lateral displacements of the source obtained with our method. For a signal to noise ratio of 300, we find that the 1-sigma error for the orthogonal displacement is $0.1 \mathrm{~cm}$, while it is negligible for the lateral displacement. These estimations of the method accuracy have been used to improve the ground calibration set-up of SuperAGILE. Searching for a good trade-off between the integration time (at the required $S / N$ ratio) and the physical quantities needed to be calibrated, we fixed a distance of $200 \mathrm{~cm}$ of the source to the detector plane. We imaged $\mathrm{Cd}^{109}$ and $\mathrm{I}^{125}$ at $\sim 200 \mathrm{~cm}$. In particular, tests with $\mathrm{Cd}^{109}$ were done from -50 up to 50 degrees with a step of 10 degrees in the experiment field of view. In this case we succeeded to obtain a good reconstruction of the source peak from -40 up to 50 degrees off-axis. The values of source distance from the detection plane as well as its angular displacement inferred from the shrinking procedure are in good agreement with the metrological measurements performed with a high precision laser tracker $(\sim 100 \mu \mathrm{m}$ accuracy). This allowed us to calibrate the conversion between detector pixel and sky degree within 2 arc minutes uncertainty (to be

compared with SuperAGILE pixel size of 6 arc minutes) and to study the transparency of materials surrounding SuperAGILE.

\section{Acknowledgments}

We are very grateful to the SuperAGILE colleagues, to the AGILE Team and to the Italian Space Agency providing a continuous support to the SuperAGILE project. In particular we thank Marco Feroci for his advices about this work and a careful reading of the manuscript.

\section{References}

1. J.J.M., in't Zand, J. Heise, and R. Jager, "The optimum open fraction of coded apertures. With an application to the wide field X-ray cameras of SAX", A \& A 288, 665-674 (1994).

2. J., Gunson, B. Polychronopulos, "Optimum design of a coded mask X-ray telescope for rocket applications",1976, MNRAS 177, 485-497 (1976).

3. J.J.M., in't Zand, "A coded-mask imager as monitor of Galactic X-ray sources", PhD Thesis (1992).

4. M. Feroci, E. Costa, P. Soffitta, E. Del Monte, G. di Persio, I. Donnarumma, I. and Y. Evangelista, M. Frutti, I. Lapshov, F. Lazzarotto, M. Mastropietro, E. Morelli, L. Pacciani, G. Porrovecchio, M. Rapisarda, A. Rubini, M. Tavani, A. Argan, "SuperAGILE: 
The hard X-ray imager for the AGILE space mission", NIM-A 581, Issue 3, 728-754 (2007).

5. I. Donnarumma, E. Costa, E. Del Monte, G. Di Persio, Y. Evangelista, M. Feroci, M. Frutti, I. Lapshov, F. Lazzarotto, A. Lo Bue, M. Mastropietro, E. Morelli, L. Pacciani, G. Porrovecchio, M. Rapisarda, P. Rossi, A. Rubini, G. Sabatino, P. Soffitta, "The onground calibrations of SuperAGILE: II. Finite distance radioactive sources", Proc. SPIE 6266, 626636 (2006).

6. M. Tavani, G. Barbiellini, A. Argan, M. Basset, F. Boffelli, A. Bulgarelli, P. Caraveo, A. Chen, E. Costa, G. De Paris, E. Del Monte, G. Di Cocco, I. Donnarumma, M. Feroci, M. Fiorini, L. Foggetta, T. Froysland, M. Frutti, F. Fuschino, M. Galli, F. Gianotti, A. Giuliani, C. Labanti, I. Lapshov, F. Lazzarotto, F. Liello, P. Lipari, F. Longo, M. Marisaldi, M. Mastropietro, E. Mattaini, F. Mauri, S. Mereghetti, E. Morelli, A. Morselli, L. Pacciani, A. Pellizzoni, F. Perotti, P. Picozza, C. Pittori, C. Pontoni, G. Porrovecchio, M. Prest, G. Pucella, M. Rapisarda, E. Rossi, A. Rubini, P. Soffitta, A. Traci, M. Trifoglio, A. Trois, E. Vallazza, S. Vercellone, A. Zambra, D. Zanello, "The AGILE mission and its scientific instrument", Proc. SPIE 6266, 626636 (2006).

7. T. M. Cannon and E. E. Fenimore, "Tomographical imaging using uniformly redundant arrays", Applied Optics 18, 1052-1057 (1979).

8. Y. Evangelista, E. Costa, E. Del Monte, G. Di Persio I. Donnarumma, M. Feroci, M. Frutti, A. Generosi, I. Lapshov, F. Lazzarotto, M. Mastropietro, E. Morelli, L. Pacciani, G. Porrovecchio, M. Rapisarda, V. Rossi-Albertini, A. Rubini, G. Sabatino and P. Soffitta, "The on-ground calibrations of SuperAGILE: I. X-ray pencil beam", Proc. SPIE 6266, 626635 (2006).

\section{APPENDIX}

We describe as the virtual detector, $\tilde{D}$, is obtained from the counts recorded by each of the pixel of the real detector $D$. As described in section 2. the $i$-th pixel of $\tilde{D}$ will collect a number of counts obtained by interpolating between the counts recorded by $[i \times m]$ and $[i \times m]+1$ pixels. We indicate them with $D(j)$ and $D(j+1)$ where $j=[i \times m]$. As it is shown in Fig. 3 they strongly depend on the projected mask tern $M(j-1), M(j), M(j+1)$ :

$$
\begin{aligned}
D(j-1) & =F\left(M_{j-1}\right)-\alpha \times F\left(M_{j-1}\right) \\
D(j) & =F\left(M_{j}\right)-\beta \times F\left(M_{j}\right)+\alpha \times F\left(M_{j-1}\right) \\
D(j+1) & =F\left(M_{j+1}\right)-\gamma \times F\left(M_{j+1}\right)+\beta \times F\left(M_{j}\right)
\end{aligned}
$$

where the function $F$ is the "transfer function" of the mask, which can assume 0 or 1 values. 
$a, b, c$ are the fraction of the source flux "lost" by $D(j-1), D(j), D(j+1)$, respectively and are so defined:

$$
\begin{aligned}
a & =(m-1)+\frac{f}{w_{\text {det }}} \times \operatorname{tg}(\theta) \\
b & =2 \times(m-1)+\frac{f}{w_{\operatorname{det}}} \times \operatorname{tg}(\theta) \\
c & =3 \times(m-1)+\frac{f}{w_{\text {det }}} \times \operatorname{tg}(\theta)
\end{aligned}
$$

where $\theta$ indicates the angle in the coding direction. The Eq. (10), (11) have to be used when $b \leq 1$.

When $b$ becomes greater than 1 , that is $\arctan \left[(1-2 \times(m-1)) \times w_{\text {det }} / f\right]<\theta<$ $\arctan \left[w_{\text {det }} / f\right], D(j)$ and $D(j+1)$ are expressed as follows:

$$
\begin{aligned}
D(j) & =\alpha \times F\left(M_{j-1}\right) \\
D(j+1) & =\beta \times F\left(M_{j}\right) .
\end{aligned}
$$

We now show the whole mathematical description for $b<1$ although we will later discuss the impact of $b \geq 1$ on the general method.

Substituting $D(j+1)$ and $D(j)$ described in Eq. (10) and Eq. (11) inside the Eq. (4), we obtain:

$$
\begin{gathered}
\tilde{D}(i)=(m-1) \times\left[F\left(M_{j+1}\right)-\gamma \times F\left(M_{j+1}\right)+\beta \times F\left(M_{j}\right)-\left(F\left(M_{j}\right)+\alpha \times F\left(M_{j-1}\right)-\beta \times\right.\right. \\
\left.\left.F\left(M_{j}\right)\right)\right]+F\left(M_{j}\right)-\beta \times F\left(M_{j}\right)+\alpha \times F\left(M_{j-1}\right)
\end{gathered}
$$

Since each element of the mask pattern could assume two possible values $(0,1)$, we have to distinguish among $2^{3}$ different combinations of the numerical tern. Then the $i$-th pixel of the virtual detector $\tilde{D}$ will collect a number of counts among the following possibilities: 


$$
\begin{aligned}
& \text { Tern values } \tilde{D}(i) \\
& 111 \frac{1}{m} \\
& 000 \quad 0 \\
& 100 \quad\left[\frac{(m-1)}{m}+\frac{f}{w_{\text {det }}} \times \frac{\operatorname{tg}(\theta)}{m}\right] \times(2-m) \\
& 010(m-1) \times\left[4 \times \frac{(m-1)}{m+2} \times \frac{f}{w_{\text {det }}} \times \frac{\operatorname{tg}(\theta)}{m}\right]+\frac{(2-m)}{m}+\frac{f}{w_{\text {det }}} \times \frac{\operatorname{tg}(\theta)}{m} \\
& 001 \quad(m-1) \times\left[\frac{(3-2 \times m)}{m}+\frac{f}{w_{\text {det }}} \times \frac{t g(\theta)}{m}\right] \\
& 110(m-1) \times\left[\frac{(2 \times m-3)}{m}+\frac{f}{w_{\text {det }}} \times \frac{\operatorname{tg}(\theta)}{m}\right]+\frac{1}{m} \\
& 011(m-1) \times\left[\frac{(m-1)}{m}+\frac{f}{w_{\text {det }}} \times \frac{\operatorname{tg}(\theta)}{m}\right]+\frac{(2-m)}{m}-\frac{f}{w_{\text {det }}} \times \frac{\operatorname{tg}(\theta)}{m} \\
& 101(m-1) \times\left[\frac{(-3 \times m+4)}{m-2} \times \frac{f}{w_{\text {det }}} \times \frac{\operatorname{tg}(\theta)}{m}\right]+\frac{(m-1)}{m}+\frac{f}{w_{\text {det }}} \times \frac{\operatorname{tg}(\theta)}{m}
\end{aligned}
$$

These expressions define the integrand functions in Eq. (5). If $\Delta \theta$ is the integration domain we remind that when $\arctan \left[(1-2 \times(m-1)) \times w_{\text {det }} / f\right]<\theta<\arctan \left[w_{\text {det }} / f\right]$ the integrand function has to change according to Eq. (12), (13). However we stress that this range of $\theta$ is $2 \times(m-1)$ wide and the contribution of the new integrand function is proportional to $m-1$, that implies a contribution $\propto(m-1)^{2}$ to the overall integral. It is then clear that when the source is placed at a distance for which $m<1.1$, this additional term could be neglected (being of order of few percent).

We note that this method is valid when the integration over $\theta$ is possible. This means that the $\triangle \theta$ integration range must be large enough to allow $a, b, c$ to cover all their definition domain, i. e. $z<N_{\text {pixel }} \times f$ where $N_{\text {pixel }}$ is the total number of detector pixels.

When $m$ in a rational number which means that $\theta$ does not vary with continuity, the summation of terms corresponding to a proper $\theta$ has to be used in place of the integral. In particular, for $m=1$ (that is for $z \rightarrow \infty$ ) one value of $\theta$ has to be considered. 


\section{List of figures}

Fig. 1 Top panel: 3D plot of bi-dimensional maximum function shape of a simulated pointsource at a distance of $250 \mathrm{~cm}$ from the detector plane; bottom panel: on the left the bidimensional maximum projected along the shrinking factor axis; on the right its projection along the shrinking base point axis.

Fig. 2 Left: the beam divergence at finite distance is shown; right: a simple scheme that summarizes the shrinking procedure; short dashed line indicates one of possible directions with respect to which source photons will be collimated.

Fig. 3 A geometrical view of the interpolation in the detector domain due to the shrinking

procedure is shown. The counts recorded by the $\mathrm{j}$-th pixel $(\tilde{D}(j))$ strongly depend on the neighbouring detector counts $(D(j-1), D(j+1)$ if $m<2) ; a, b, c$ represent the fraction of source flux "lost" passing through the mask pattern numerical tern $M(j-1), M(j), M(j+1)$, respectively.

Fig. 4 Solid line: reconstructed sky image of point source at infinite distance (obtained using the standard deconvolution method); dot: focused image of same source simulated at finite distance ( say $\mathrm{z}=250 \mathrm{~cm}$ ); dashed line: the same for a $5 \mathrm{~mm}$ extended source.

Fig. 5 The left panel shows the image of a point source simulated at $z+f=250 \mathrm{~cm}$ obtained with the standard deconvolution method; the right panel shows the image obtained for the same source after the application of the shrinking procedure.

Fig. 6 Reconstructed images for $\mathrm{Cd}^{109}$ at 2 meters from the detection plane from -40 up to 50 degrees off-axis. The angular size of each bin is 3 arc minutes (one SuperAGILE sky pixel). Fig. $7 \mathrm{Cd}^{109}$ at 2 meters from the detection plane at 0 and 30 degrees off-axis in the total field of view of SuperAGILE.

Fig. 8 SuperAGILE pixel-angle relation (for each of the four detector units). 

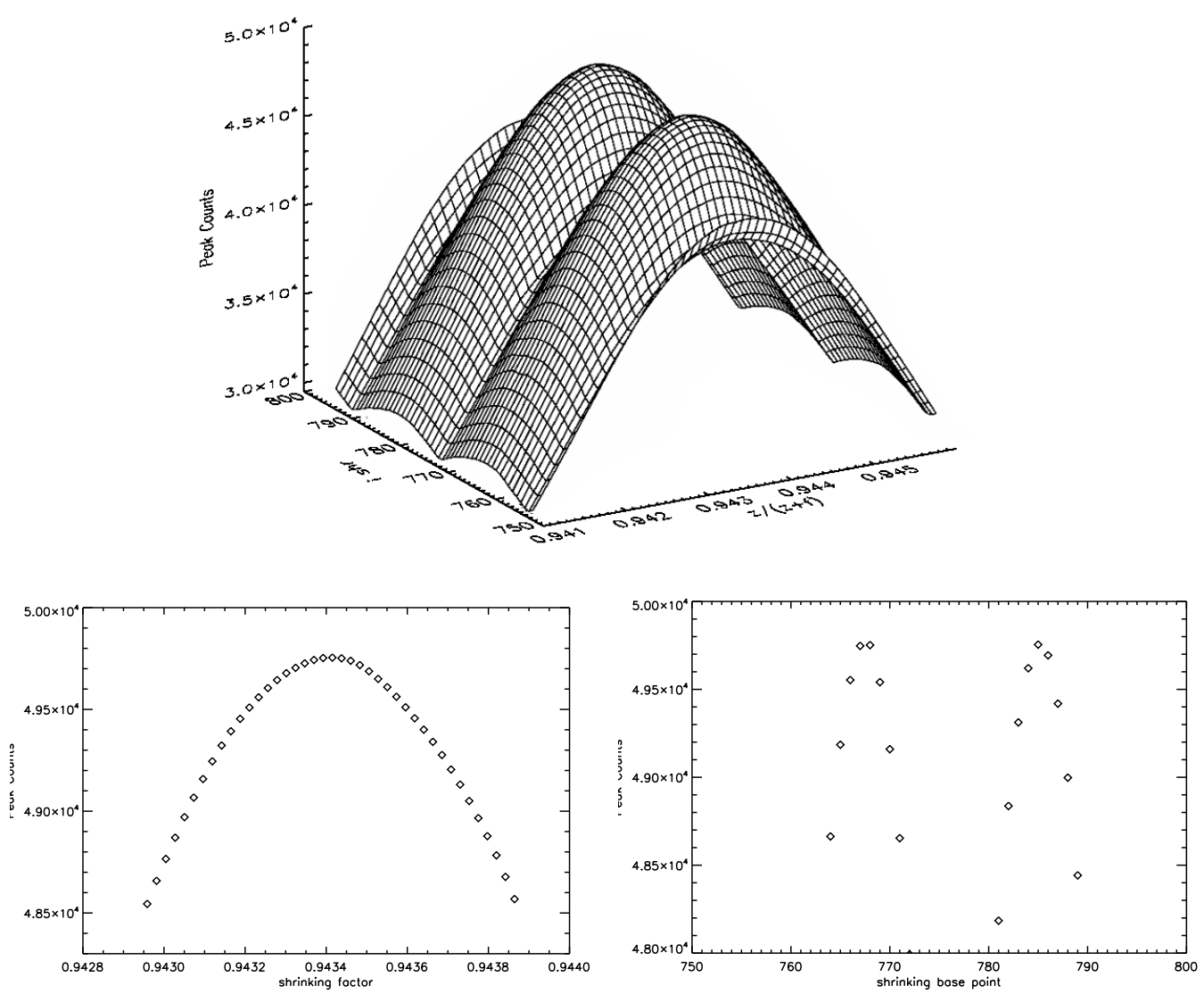

Fig. 1. Top panel: 3D plot of bi-dimensional maximum function shape of a simulated point-source at a distance of $250 \mathrm{~cm}$ from the detector plane; bottom panel: on the left the bi-dimensional maximum projected along the shrinking factor axis; on the right its projection along the shrinking base point axis. 


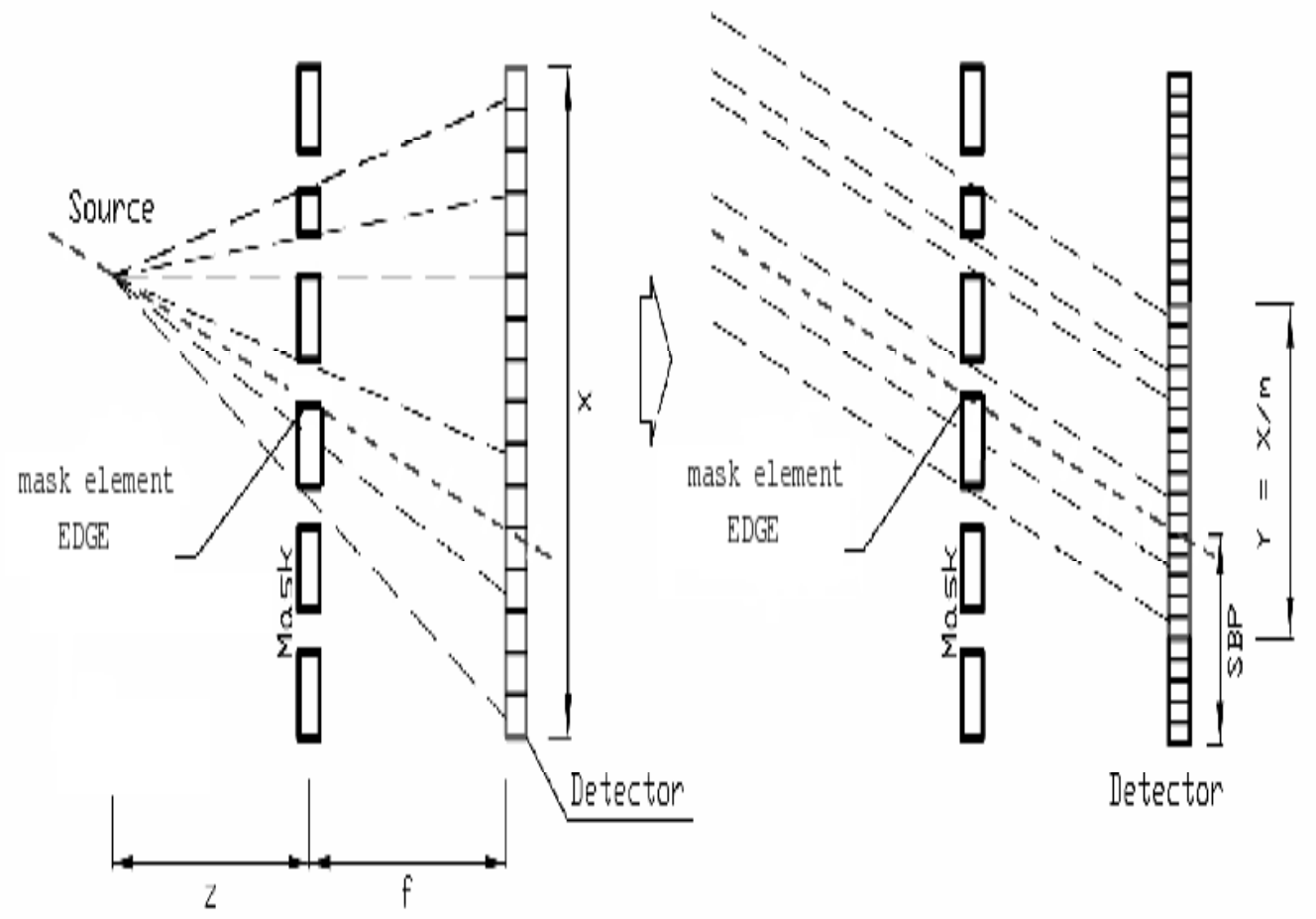

Fig. 2. Left: the beam divergence at finite distance is shown; right: a simple scheme that summarizes the shrinking procedure; short dashed line indicates one of possible directions with respect to which source photons will be collimated. 


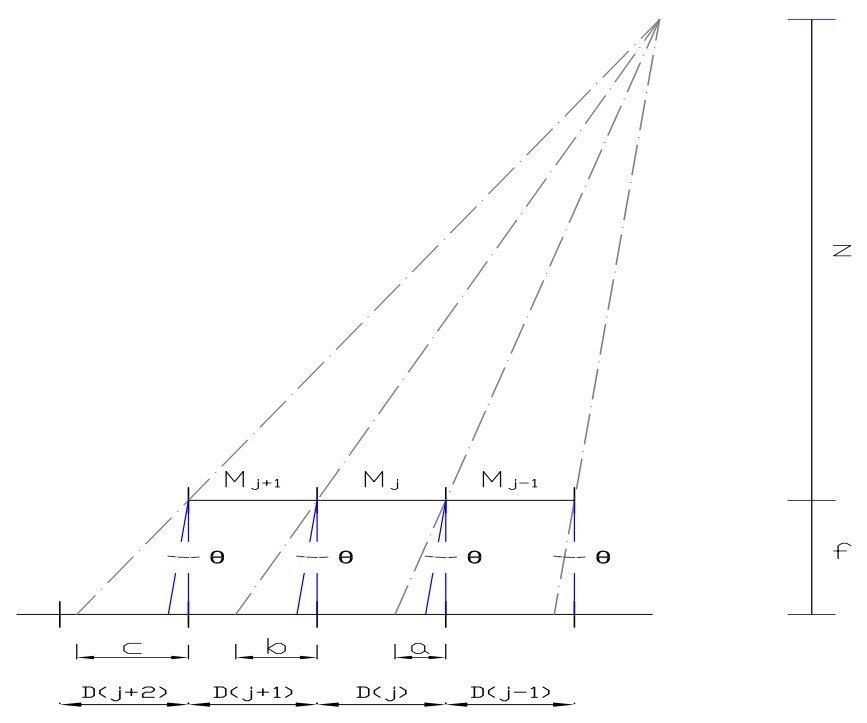

Fig. 3. A geometrical view of the interpolation in the detector domain due to the shrinking procedure is shown. The counts recorded by the j-th pixel $(\tilde{D}(j))$ strongly depend on the neighbouring detector counts $(D(j-1), D(j+1)$ if $m<2) ; a, b, c$ represent the fraction of source flux "lost" passing through the mask pattern numerical tern $M(j-1), M(j), M(j+1)$, respectively. 


\begin{tabular}{ccc}
\hline$z+f(\mathrm{~cm})$ & peak counts & $\triangle \mathrm{f}(\mu \mathrm{m})$ \\
\hline & & \\
100 & 0.84 & 165 \\
130 & 0.83 & 110 \\
150 & 0.83 & 90 \\
180 & 0.83 & 80 \\
200 & 0.82 & 78 \\
230 & 0.82 & 75 \\
250 & 0.82 & 75 \\
270 & 0.82 & 60 \\
300 & 0.82 & 60 \\
\hline
\end{tabular}

Table 1. The reconstructed peak counts as a function of source distance to the detector plane; third column: the mask-detector separation error inferred by the statistical error $\Delta \mathrm{z}$ as a function of $z+f$.

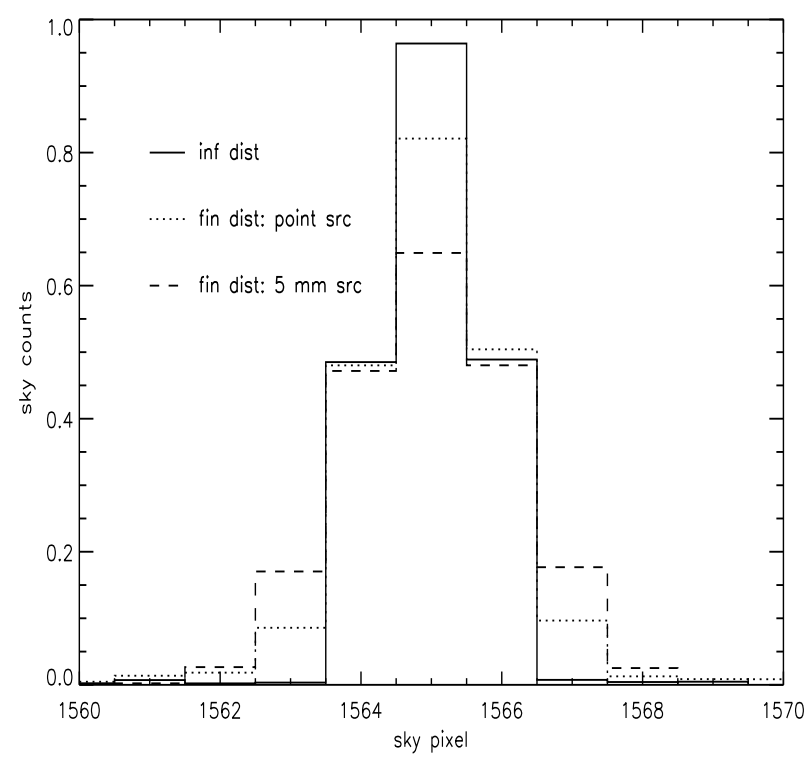

Fig. 4. Solid line: reconstructed sky image of point source at infinite distance (obtained using the standard deconvolution method); dot: focused image of same source simulated at finite distance ( say $\mathrm{z}=250 \mathrm{~cm}$ ); dashed line: the same for a $5 \mathrm{~mm}$ extended source. 

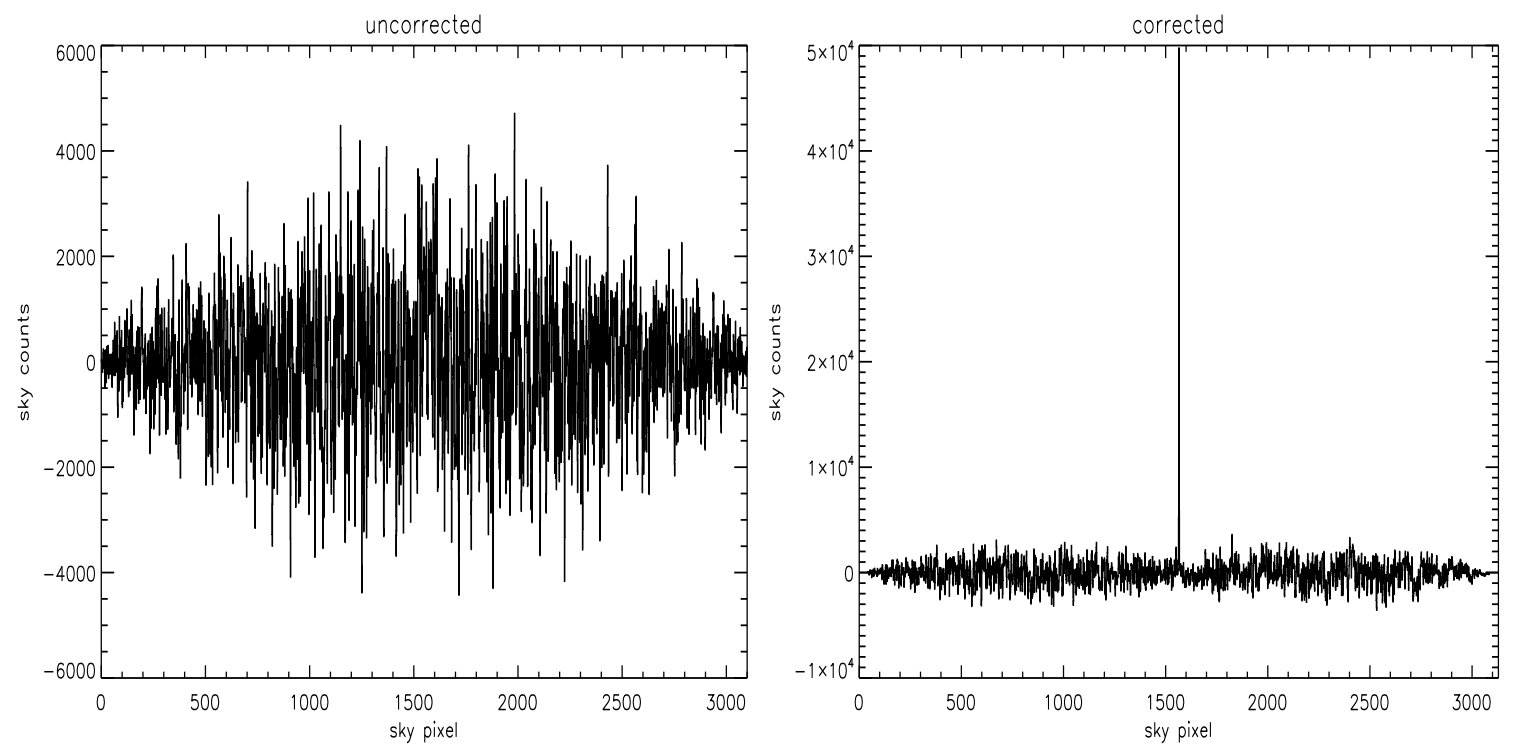

Fig. 5. The left panel shows the image of a point source simulated at $z+f=250$ $\mathrm{cm}$ obtained with the standard deconvolution method; the right panel shows the image obtained for the same source after the application of the shrinking procedure. 

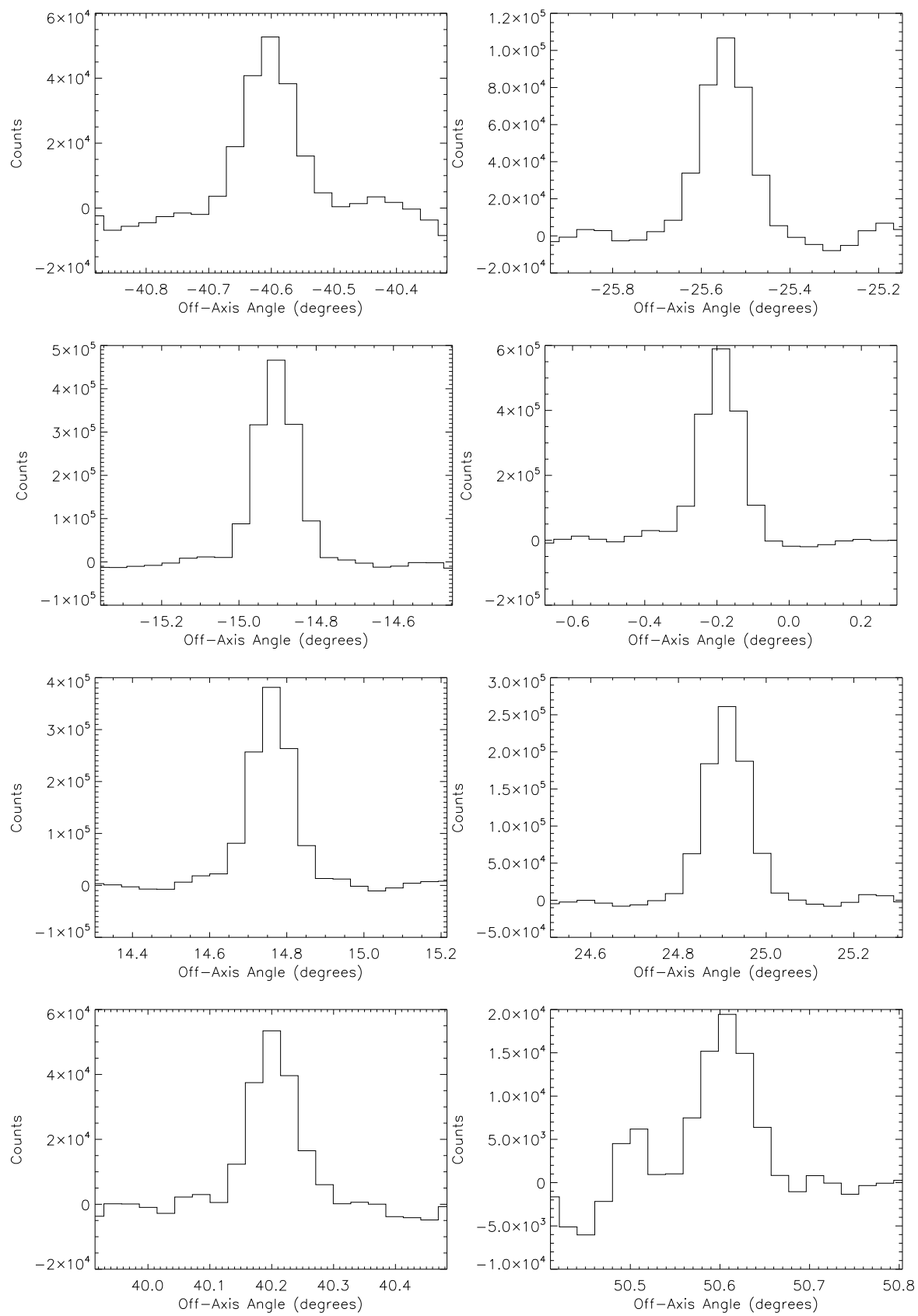

Fig. 6. Reconstructed images for $\mathrm{Cd}^{109}$ at 2 meters from the detection plane from -40 up to 50 degrees off-axis. The angular size of each bin is 3 arc minutes (one SuperAGILE sky pixel). 

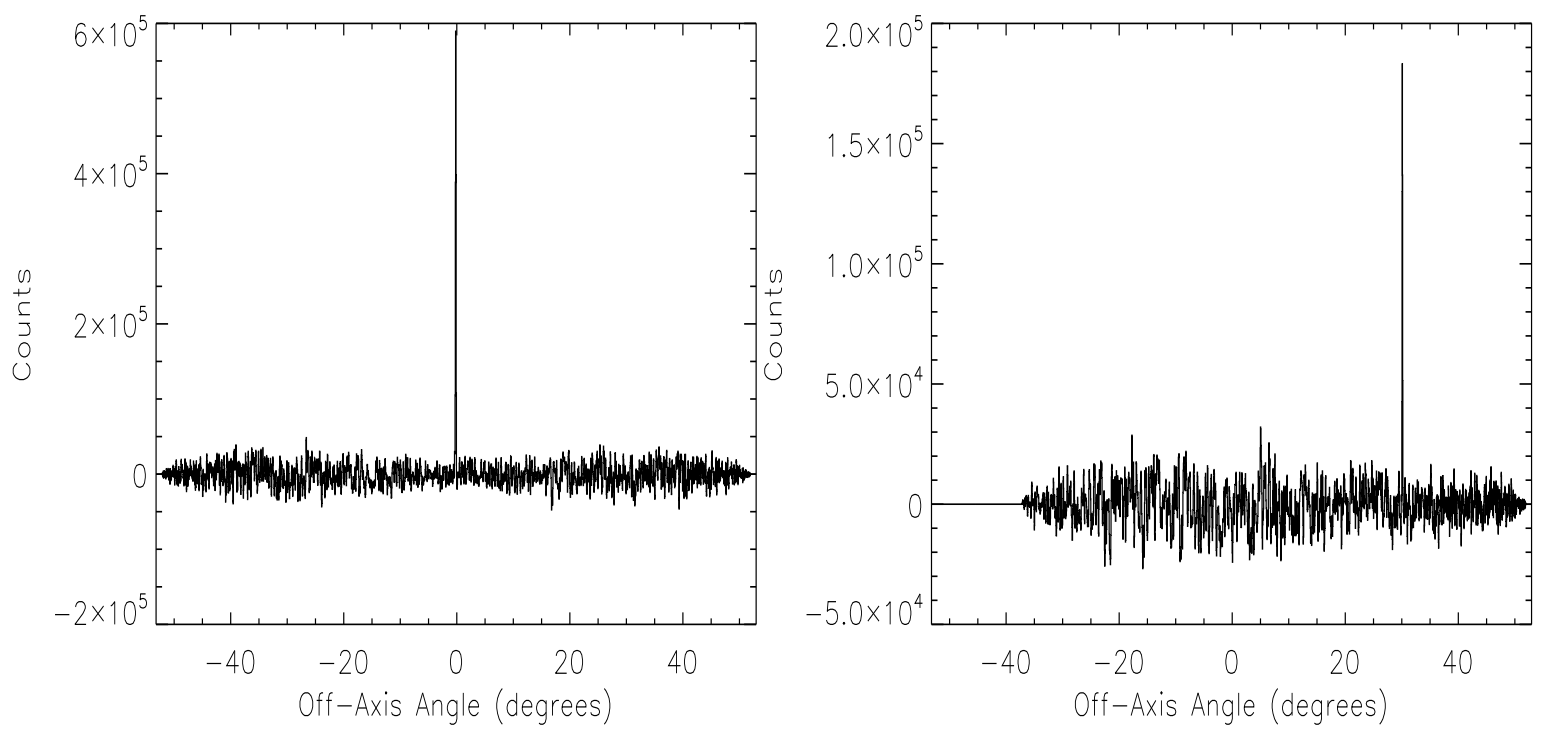

Fig. 7. $\mathrm{Cd}^{109}$ at 2 meters from the detection plane at 0 and 30 degrees off-axis in the total field of view of SuperAGILE. 

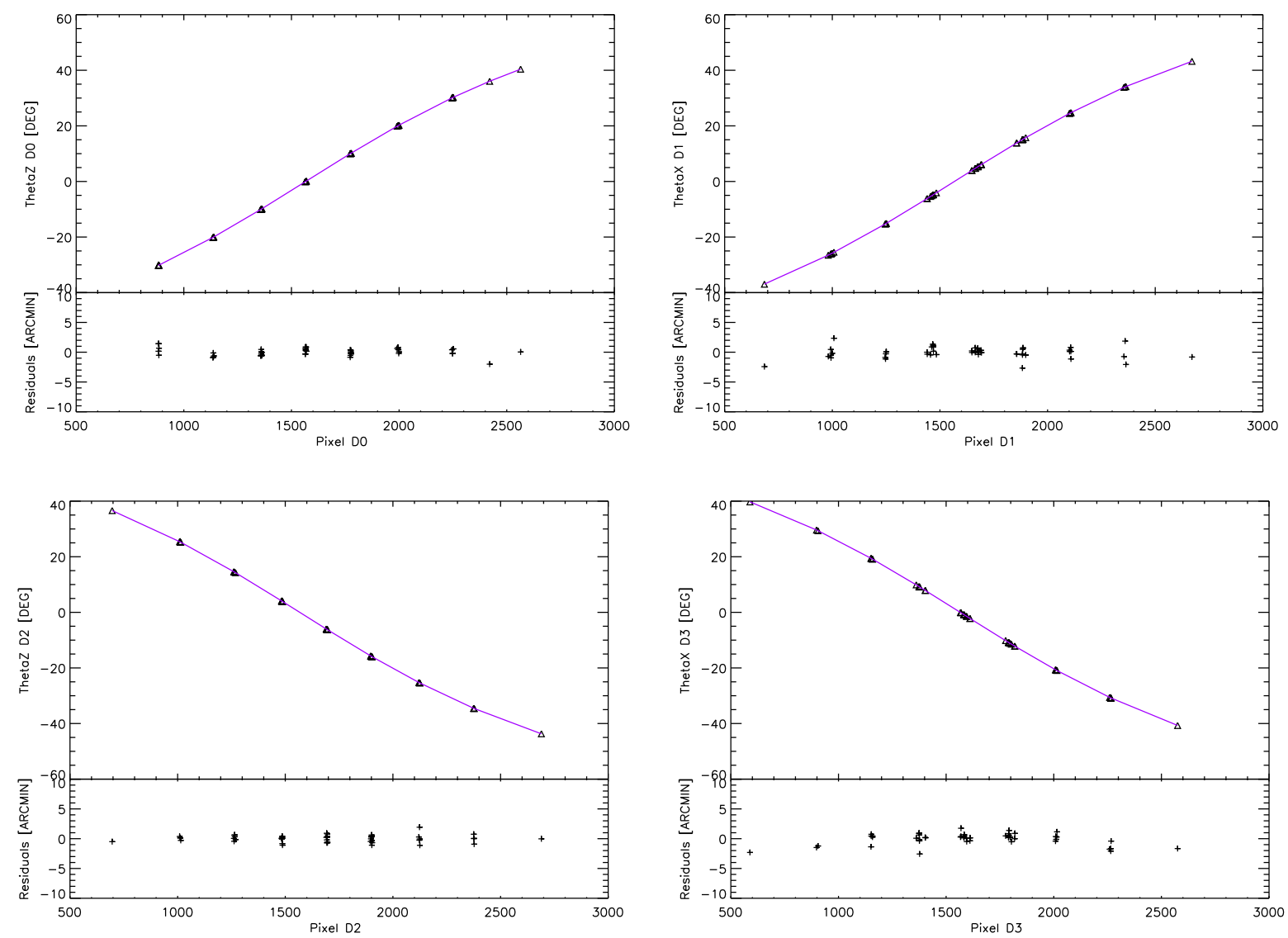

Fig. 8. SuperAGILE pixel-angle relation (for each of the four detector units). 